J. Dairy Sci. 97:6129-6134

http://dx.doi.org/10.3168/jds.2014-8344

(C) American Dairy Science Association ${ }^{\circledR}, 2014$.

\title{
Short communication: Effects of lactose and milk on the expression of biofilm-associated genes in Staphylococcus aureus strains isolated from a dairy cow with mastitis
}

\author{
Ting Xue, ${ }^{1}$ Xiaolin Chen, and Fei Shang \\ School of Life Sciences, Anhui Agricultural University, Hefei, Anhui 230036, China
}

\begin{abstract}
Staphylococcus aureus is the main etiological organism responsible for bovine mastitis. The ability of $S$. aureus to form biofilms plays an important role in the pathogenesis of mastitis. Biofilm formation in S. aureus is associated with the production of polysaccharide intercellular adhesin (PIA) protein and several other proteins. Several environmental factors, including glucose, osmolarity, oleic acid, temperature, and anaerobiosis, have been reported to affect biofilm formation in S. aureus. This study investigated the influence of lactose and milk on the biofilm formation capacity of 2 clinical bovine isolates of $S$. aureus. We found that lactose increased biofilm formation predominantly by inducing PIA production, whereas milk increased biofilm formation through PIA as well as by increasing the production of other biofilm-associated proteins, which might be mediated by the transcriptional regulators intercellular adhesion regulator $(i c a R)$ and repressor of biofilm $(r b f)$.
\end{abstract}

Key words: Staphylococcus aureus, mastitis, biofilm, lactose, milk

\section{Short Communication}

Mastitis is an important disease and is the most common cause of death in adult dairy cows (Booth, 1997). The economic losses arising from bovine mastitis treatment and death as well as due to decreased milk production are significant. Staphylococcus aureus is postulated to be an important etiological organism responsible for this disease (AHVLA, 2001). Staphylococcus aureus virulence in mastitis predominantly relies on the production of several exotoxins and surface proteins, the ability to survive within host phagocytic cells, and the ability to form biofilms that are composed of multilayered cell clusters embedded in a slime matrix (Aguilar et al., 2001; Takeuchi et al., 2001). Biofilm formation

Received May 9, 2014

Accepted July 9, 2014

${ }^{1}$ Corresponding author: xuet@ahau.edu.cn facilitates the adherence and colonization of $S$. aureus on mammary gland epithelium (Baselga et al., 1993). In addition, biofilm formation in mastitis isolates of $S$. aureus is associated with reduced susceptibility to antibiotics and evasion of the immunological defenses of the cow immune system (Amorena et al., 1999; Aguilar et al., 2001; Vasudevan et al., 2003). The structure of the biofilm protects bacteria against high antimicrobial concentrations and phagocytosis, aiding in survival in hostile environments within the host (Fox et al., 2005;

Table 1. Oligonucleotide primers used in this study

\begin{tabular}{ll}
\hline Primer name & Oligonucleotide $\left(5^{\prime}-3^{\prime}\right)$ \\
\hline rt-icaA-f & TTTCGGGTGTCTTCACTCTAT \\
rt-icaA-r & CGTAGTAATACTTCGTGTCCC \\
rt-icaB-f & CCTATCCTTATGGCTTGATGA \\
rt-icaB-r & CATTGGAGTTCGGAGTGA \\
rt-icaC-f & TACTGACAACCTGAATTACA \\
rt-icaC-r & AATAGCCATACCATTGACCTAA \\
rt-icaD-f & CCAGACAGAGGGAATACC \\
rt-icaD-r & AAGACACAAGATATAGCGATAAG \\
rt-icaR-f & TTATCTAATACGCCTGAGGAAT \\
rt-icaR-r & GGATGCTTTCAAATACCAACT \\
rt-rbf-f & CGATATGCGTATTATGGTGATT \\
rt-rbf-r & AAGTAAGTGGAATTGTGATGAC \\
rt-bap-f & ATACTGATGGCGATGGTA \\
rt-bap-r & ACTGTGTCTTCTGTTGTAAT \\
rt-aap-f & AACAACAACAACACCAACT \\
rt-aap-r & GCCACCATAATGAACGATT \\
rt-sigB-f & ATTGCTTCTAACACTTCTTCTTCT \\
rt-sigB-r & CGATGAACTAACCGCTGAAT \\
rt-sarA-f & GTTATCAATGGTCACTTATGC \\
rt-sarA-r & CTTGTGGTTGTTTGTAGTTT \\
rt-sarX-f & AACATTGCTTGGCTTCTAT \\
rt-sarX-r & AATCTAGCTCATCCATTGC \\
rt-sarZ-f & AACACTGACACCATTACTAA \\
rt-sarZ-r & GATGCTTCTCGTTCTGAA \\
rt-agrA-f & GCAGTAATTCAGTGTATGTTCA \\
rt-agrA-r & TATGGCGATTGACGACAA \\
rt-clpP-f & CTCAAGGACAAGCAACTG \\
rt-clpP-r & ATCACTTCATCAATTAAGCCATA \\
rt-arlR-f & GACAACAACAATCTACACCTAT \\
rt-arlR-r & TTCTTCAATATCAAACGGCTTA \\
rt-saeR-f & ATCGTGGATGATGAACAA \\
rt-saeR-r & GTCGTAACCATTAACTTCTG \\
rt-rsbU-f & GCACAACCTAATATGAATGG \\
rt-rsbU-r & TAACAGAAGCGGCTACTA \\
rt-abfR-f & AAGACTTGAGCAATCTGG \\
rt-abfR-r & TGTGGTAAGCAACTTGAA \\
\hline rf = forward; r $=$ reverse. & \\
&
\end{tabular}




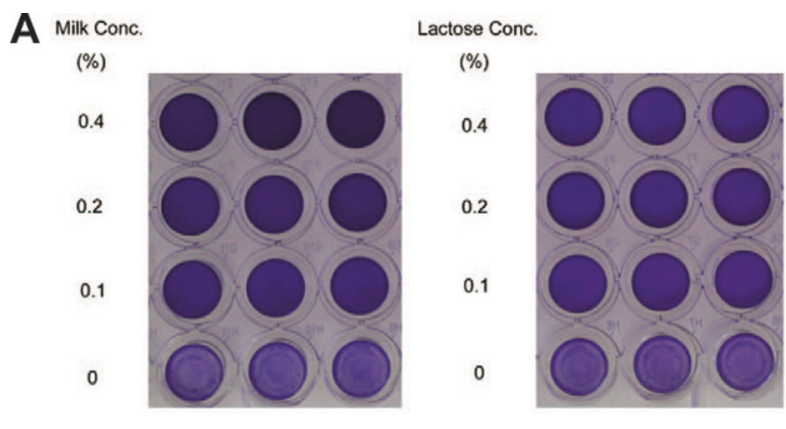

S. aureus DCM1

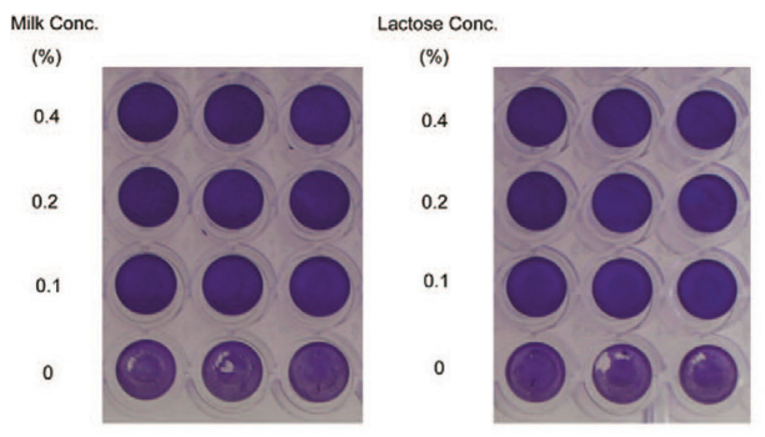

S. aureus DCM2

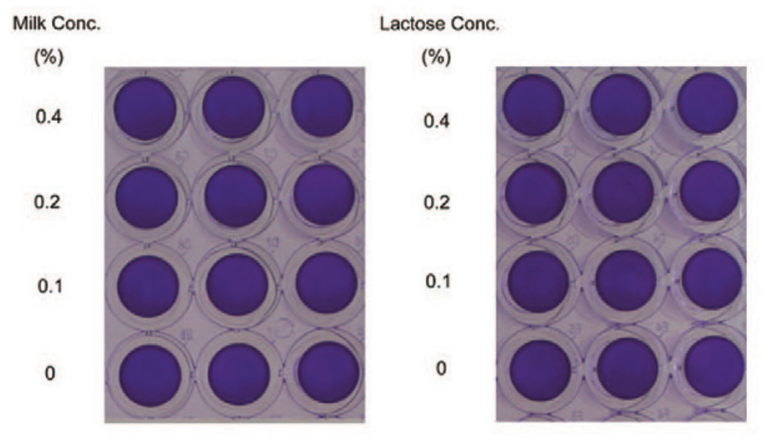

S. aureus NCTC8325

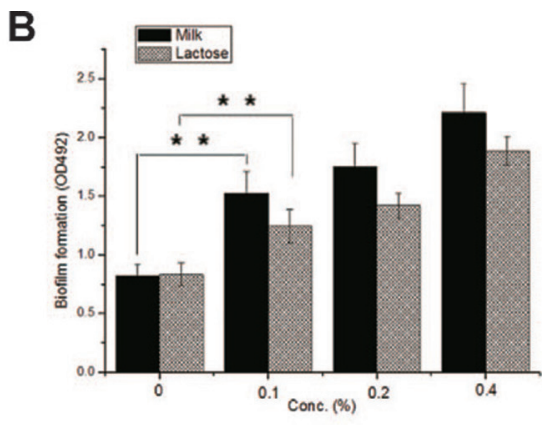

S. aureus DCM1

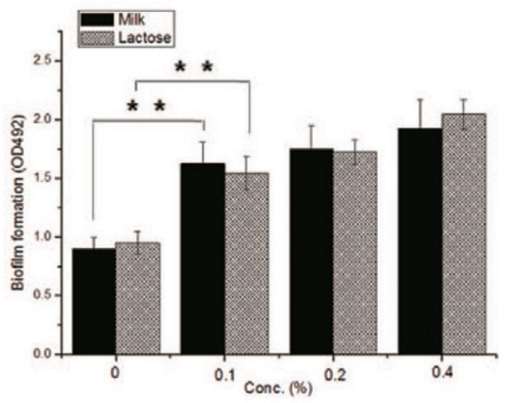

S. aureus DCM2

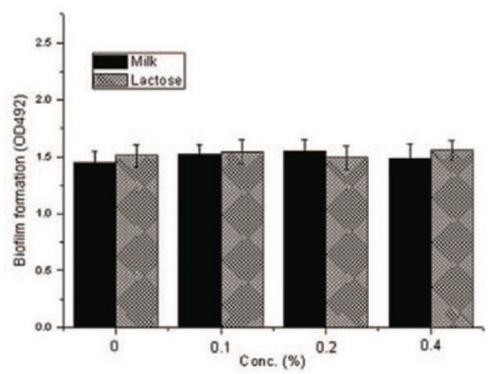

S. aureus NCTC8325

Figure 1. Effect of milk or lactose on biofilm formation in Staphylococcus aureus DCM1, S. aureus DCM2, and S. aureus NCTC8325. Biofilm growth of $S$. aureus strains cultured with milk or lactose at different concentrations (Conc.; 0, 0.1, 0.2, and 0.4\%) in 96-well plates for 16 h under aerobic conditions. (A) The photographs of the 96-well plates after staining with crystal violet. (B) The cells that adhered to the plate after staining with crystal violet were measured by the optical density at $492 \mathrm{~nm}$ (OD492). Error bars indicate SD. The results represent means of 3 independent experiments. ${ }^{* *} P<0.01$. Color version available in the online PDF.

Melchior et al., 2006). Therefore, biofilm-forming isolates of $S$. aureus can cause persistent infection in the bovine mammary gland, which is difficult to eradicate. A high proportion of biofilm-positive strains were characterized in bovine isolates of $S$. aureus (Oliveira et al., 2006).

Scanning electron microscopy studies indicate a 2 -step process of biofilm formation in which the bacteria first adhere to a surface and then multiply to form a multilayered biofilm that is surrounded by a slimy matrix (Peters et al., 1981). The slimy substance has been characterized as polysaccharide intercellular adhesin (PIA), which is generated by an intercellular adhesion (ica) locus consisting of 4 open reading frames: $i c a A$, icaD, icaB, and icaC. A transcriptional regulator gene, intercellular adhesion regulator $(\boldsymbol{i c a R})$, which is divergently transcribed from the $i c a$ operon, has been identified as the repressor of the ica operon (McKenney 
et al., 1998; Cramton et al., 1999). In addition, several other factors related to biofilm formation have been identified, such as accumulation-associated protein (AAP), staphylococcal surface protein (SSP1), and biofilm-associated protein (Bap; Cucarella et al., 2001; Götz, 2002).

Several environmental factors, including glucose, osmolarity, oleic acid, temperature, and anaerobiosis, have been reported to affect biofilm formation in $S$. aureus (Campbell et al., 1983; Mack et al., 1992; Rachid et al., 2000; Cramton et al., 2001). Some previous studies have regarded the influence of milk in biofilm production by $S$. aureus mastitis isolates (Amorena et al., 1999; Oliveira et al., 2006). It is known that lactose is the unique saccharide in the mammalian mammary gland. However, whether lactose affects biofilm-associated genes of $S$. aureus remains unclear. This study investigated whether lactose or milk influence biofilm formation and biofilm-associated genes of 2 clinical bovine isolates of $S$. aureus.

Staphylococcus aureus strains DCM1 and DCM2 were isolated from a dairy cow with mastitis. Strains were grown in tryptic soy broth (TSB) containing $0.25 \%$ glucose media (Oxoid Ltd., Basingstoke, UK). Biofilm quantification was performed as described previously and modified as follows (Ziebuhr et al., 1997; Pozzi et al., 2012). Staphylococcus aureus strains were grown for $16 \mathrm{~h}$ in TSB, and diluted 1:100 in fresh TSB. Milk or lactose was added to the TSB medium with diluted cultures at different concentrations. Cultures were transferred to sterile 96-well flat-bottomed tissue culture plates and incubated for $16 \mathrm{~h}$ at $37^{\circ} \mathrm{C}$. Adherent bacteria were stained with crystal violet, and excess stain was gently rinsed with water. After drying, the stained biofilm was read using a MicroELISA autoreader (Thermo Scientific, Pittsburgh, PA) at a wavelength of $492 \mathrm{~nm}$ in single wavelength mode.

Overnight, $S$. aureus cultures were diluted 1:100 in Luria-Bertani media and grown to late exponential phase (optical density at $600 \mathrm{~nm}=2.1$ ). Cells were collected and resuspended in Tris-EDTA buffer (pH 8.0) containing $10 \mathrm{~g}$ of lysozyme/L and $40 \mathrm{mg}$ of lysostaphin/L. After incubation for $5 \mathrm{~min}$ at $37^{\circ} \mathrm{C}$, total RNA was extracted from cells using the TRIzol reagent (Ambion
A

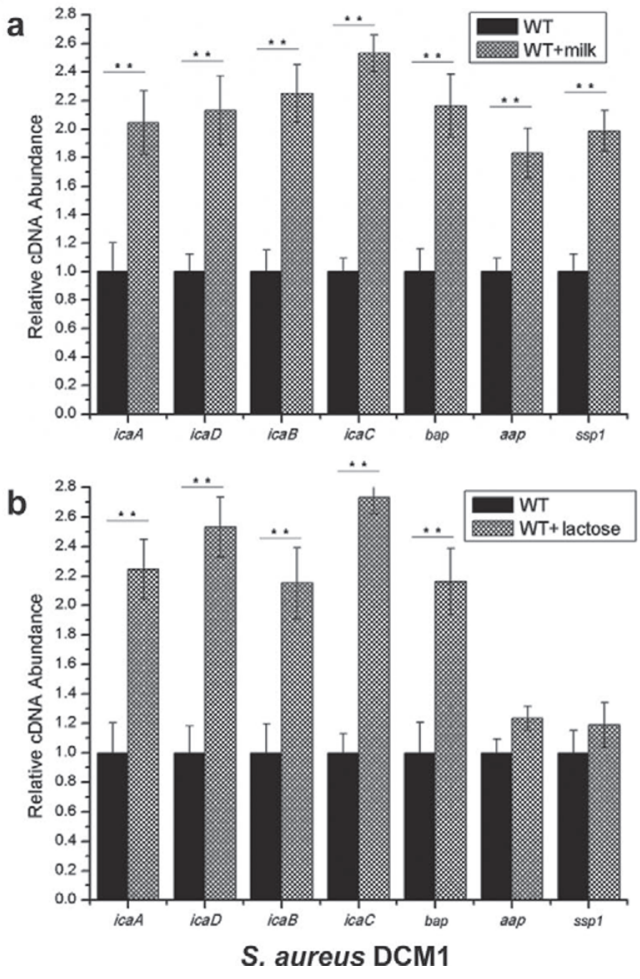

B
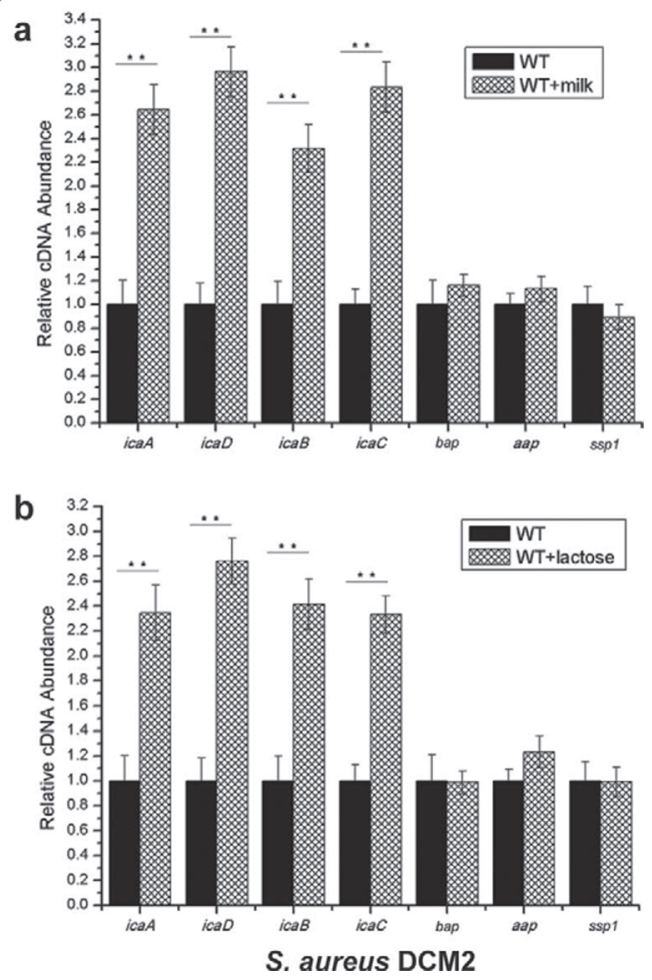

Figure 2. Comparative measurement of transcription of the biofilm-associated genes. (A) Relative transcript levels of intercellular adhesion (ica), biofilm-associated protein ( $b a p)$, accumulation-associated protein (aap), and staphylococcal surface protein (ssp1) in Staphylococcus aureus DCM1 (WT, wild-type) and S. aureus DCM1 with milk (a) and that in $S$. aureus DCM1 and S. aureus DCM1 with lactose (b), were determined by real-time reverse-transcription PCR (RT-PCR). (B) Relative transcript levels of ica, bap, aap, and ssp1 in S. aureus DCM2 and S. aureus DCM2 with milk (a) and that in $S$. aureus DCM2 and $S$. aureus DCM2 with lactose (b), were determined by real-time RT-PCR. Error bars indicate SD. The results represent means of 3 independent experiments. ${ }^{* *} P<0.01$. 
Inc., Austin, TX), and residual DNA was removed using DNase [TaKaRa Biotechnology (Dalian) Co. Ltd., Dalian, China]. Quantitative reverse-transcription PCR (RT-PCR) was performed using the PrimeScript 1st Strand cDNA Synthesis Kit, SYBR Premix Ex Taq [TaKaRa Biotechnology (Dalian) Co. Ltd.], and a StepOne Real-Time PCR System (Applied Biosystems LLC, Carlsbad, CA). Cycling conditions were as follows: $95^{\circ} \mathrm{C}$ for $30 \mathrm{~s}$ and then 40 cycles of $95^{\circ} \mathrm{C}$ for $5 \mathrm{~s}$ and $60^{\circ} \mathrm{C}$ for $30 \mathrm{~s}$. The quantity of cDNA measured by real-time PCR was normalized to the abundance of $16 \mathrm{~S}$ cDNA. Primers were designed according to the genome sequence information of $S$. aureus strains NCTC8325 and N315 using Primer software (Premier Biosoft International, Palo Alto, CA; Table 1).

To examine the influence of lactose on biofilm formation capacity of the $S$. aureus isolates, biofilm assays were performed. Lactose is one of the components in milk. However, milk contains other substances, including proteins, fats, and mineral salts. Therefore, we tested the effects of lactose as well as the effects of milk on biofilm formation capacity of $3 \mathrm{~S}$. aureus isolates ( 2 bovine isolates and 1 human isolate). The $3 S$. aureus strains were grown in TSB for $16 \mathrm{~h}$ and diluted 1:100 in fresh TSB. Milk or lactose was added to the TSB medium to final concentrations of $0,0.1$, 0.2 , and $0.4 \%$. Cultures were transferred to sterile $96-$ well flat-bottomed tissue culture plates and were incubated for $16 \mathrm{~h}$ at $37^{\circ} \mathrm{C}$. After crystal violet staining, we observed biofilm biomass in the 96-well plates. As shown in Figure 1A, when lactose or milk was added to wells containing the bovine isolates DCM1 and DCM2, we observed significant increases in biofilm formation; however, the addition of lactose or milk did not affect biofilm formation in wells containing the human isolate S. aureus NCTC8325 (Figure 1A). In addition, the quantity of biofilm formation was further tested using a MicroELISA autoreader. We found that biofilm quantity increased by varying degrees with increased concentrations of milk or lactose in strains DCM1 and DCM2 (Figure 1B). Because the biomass of biofilm that was altered by adding milk and lactose was almost equivalent, we speculate that it is the lactose in milk that primarily affects biofilm formation.

To investigate the mechanism of how lactose and milk regulate biofilm formation in $S$. aureus isolates, real-time RT-PCR experiments were performed to evaluate the transcript levels of biofilm-associated genes influenced by lactose and milk. We first examined the $i c a$ operon, which encodes the PIA synthetases. The transcript levels of $i c a A, i c a B, i c a C$, and $i c a D$ in both bovine isolates were increased upon the addition of milk or lactose (Figure 2). These data indicate that in both bovine isolates, lactose and milk induced biofilm
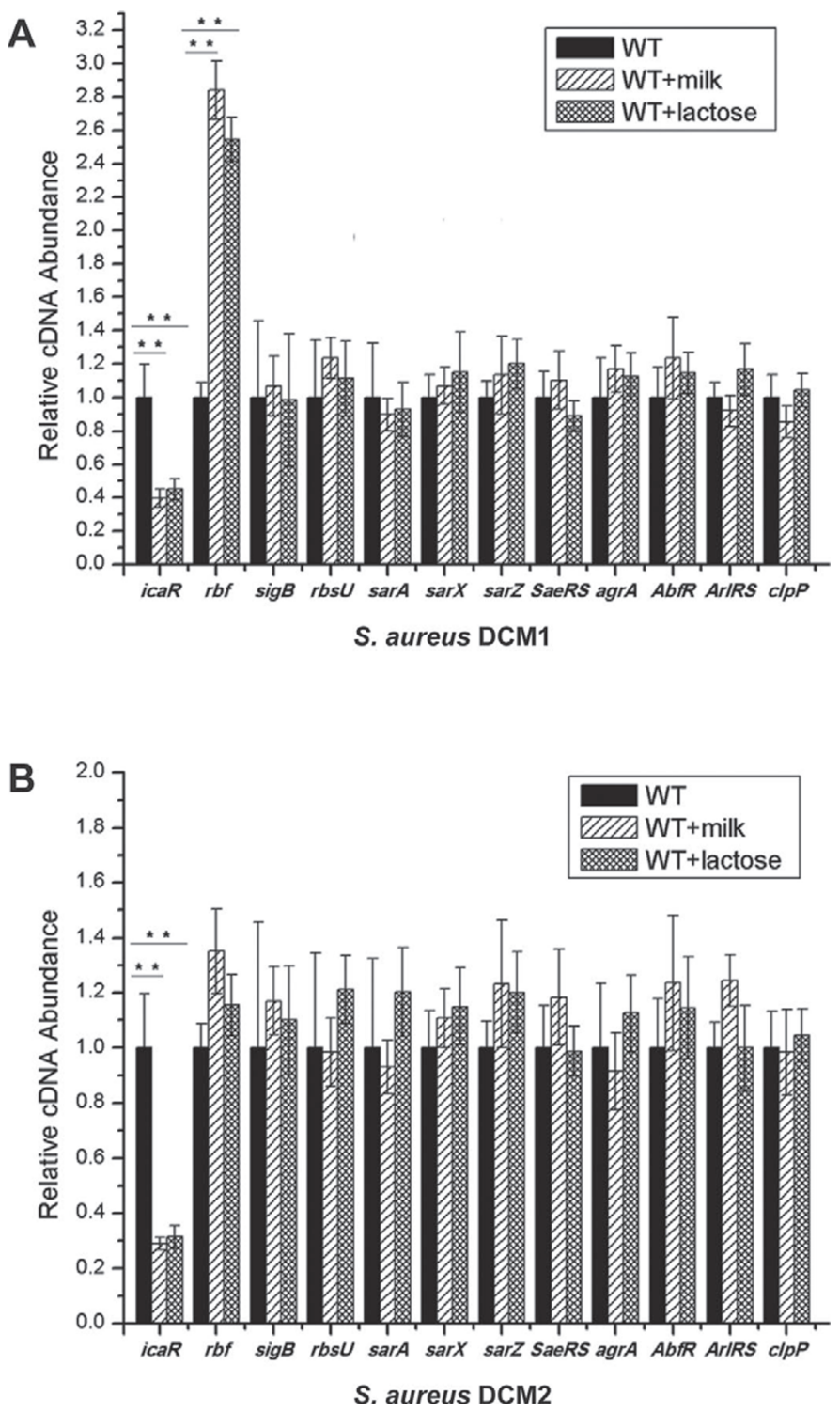

Figure 3. Comparative measurement of transcription of the biofilm regulator genes. (A) Relative transcript levels of repressor of biofilm $(r b f), \operatorname{sig} B$, ribose transporter $(r b s U)$, systemic acquired resistance A $(\operatorname{sar} A), \operatorname{sar} X, \operatorname{sar} Z$, caseinolytic mitochondrial matrix peptidase proteolytic subunit $(\operatorname{clp} P)$, accessory gene regulator A $(\operatorname{agr} A)$, arabinofuranosidase two-component system response regulator $(a b f R)$, arlR $S$, and saeRS were determined by real-time reverse-transcription PCR (RT-PCR) in Staphylococcus aureus DCM1 (WT, wild-type), S. aureus DCM1 with milk, and $S$. aureus DCM1 with lactose. (B) Relative transcript levels of $r b f, \operatorname{sig} B, \operatorname{rbs} U, \operatorname{sar} A, \operatorname{sar} X, \operatorname{sar} Z, \operatorname{clp} P, \operatorname{agr} A, a b f R$, $\operatorname{arlRS}$, and saeRS were determined by real-time RT-PCR in $S$. aureus DCM2, S. aureus DCM2 with milk, and S. aureus with lactose. Error bars indicate SD. The results represent means of 3 independent experiments. ${ }^{* *} P<0.01$.

formation by increasing PIA production. Additionally, according to previous studies, several other genes that encode proteins, such as bap, aap, and ssp1, are associated with the biofilm formation process (Cucarella et al., 2001; Götz, 2002). Thus, we also performed real-time 
Table 2. Effects of lactose and milk on the expression of biofilm-associated genes in Staphylococcus aureus DCM1, DCM2, and NCTC8325

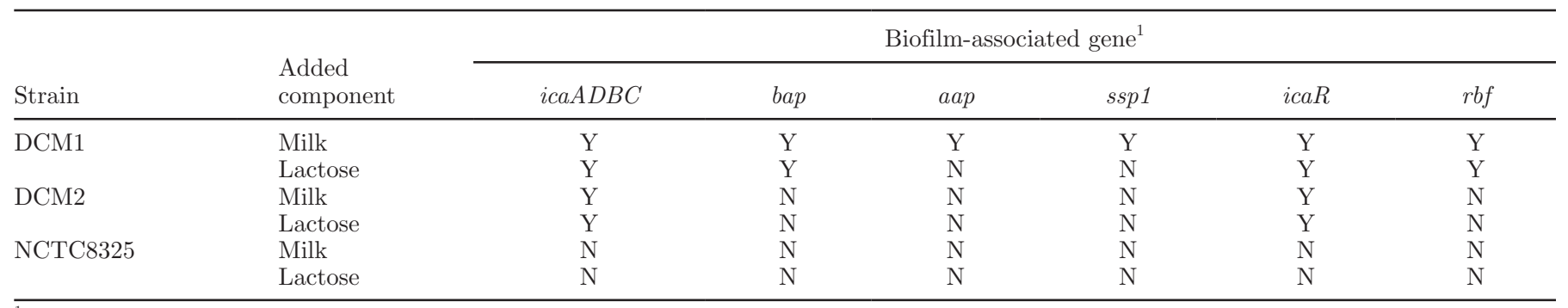

${ }^{1} i c a=$ intercellular adhesion; $b a p=$ biofilm-associated protein; $a a p=$ accumulation-associated protein; ssp1 = staphylococcal surface protein; $i c a R=$ intercellular adhesion regulator; $r b f=$ repressor of biofilm ; $\mathrm{Y}=$ yes; $\mathrm{N}=$ no.

RT-PCR experiments to measure the transcript levels of these genes. As shown in Figure 2Aa, bap, aap, and ssp1 transcription in $S$. aureus DCM1 was apparently increased upon the addition of milk compared with the wild type. However, only bap transcription increased by adding lactose to the culture media. The transcription of aap and ssp1 were not affected upon the addition of lactose compared with the wild-type strain in $S$. aureus DCM1 (Figure 2Ab). These results indicate that in $S$. aureus DCM1, lactose, and milk affect bap transcription. In addition, it is interesting that the effect of milk on $a a p$ and ssp 1 transcription differed from that of lactose. It is likely that additional components in milk affected aap and ssp1 transcription. However, in S. aureus DCM2, bap, aap, and ssp1 transcription did not appear to change upon the addition of milk (Figure $2 \mathrm{Ba}$ ) or lactose (Figure 2Bb).

To further investigate how lactose and milk regulate biofilm-associated genes, we examined the transcript levels of regulatory genes that have been identified to be involved in biofilm formation. Due to changes in the transcription of $i c a A B C D$, the transcript level of $i c a R$ was examined. As expected, ica $R$ transcription decreased by adding milk or lactose to the wild-type culture medium in $S$. aureus DCM1 (Figure 3A) and $S$. aureus DCM2 (Figure 3B), indicating that both milk and lactose influence the ica operon through icaR. To find the intermediates of the milk- or lactose-regulating $i c a R$ pathway, the transcript levels of known biofilm regulator genes [repressor of biofilm $(r b f)$, sigB, ribose transporter $(r b s U)$, systemic acquired resistance A $(\operatorname{sar} A), \operatorname{sar} X, \operatorname{sar} Z$, caseinolytic mitochondrial matrix peptidase proteolytic subunit $(\operatorname{clp} P)$, accessory gene regulator A ( agr $A)$, arabinofuranosidase two-component system response regulator $(a b f R)$, arlRS, and saeRS] were tested. Among these factors, only $r b f$ was affected by milk and lactose in S. aureus DCM1; however, in $S$. aureus DCM2, no obvious changes occurred in the transcript levels of any of these genes, suggesting that lactose may influence biofilm formation via additional mechanisms.
Previous studies have shown that $S$. aureus biofilm composition as well as the influencing factors and regulatory mechanism of biofilm formation are strain dependent (Götz, 2002). In our study, milk and lactose did not obviously affect biofilm formation in $S$. aureus NCTC8325, which is a human isolate. However, the biofilm formation capacity of the 2 bovine isolates was dramatically increased in vitro upon the addition of milk or lactose to the culture media, indicating that the bovine isolates may have evolved to utilize milk ingredients to increase biofilm formation after longterm infection in dairy cows. In addition, we found that lactose increased biofilm formation predominantly by enhancing PIA production, whereas milk increased biofilm formation through PIA as well as by increasing production of other biofilm-associated proteins, which might be mediated by $i c a R$ and $r b f$ (Table 2). These findings provide new clues regarding the prevention and treatment of mastitis caused by biofilm-positive $S$. aureus in dairy cows.

\section{ACKNOWLEDGMENTS}

This work was supported by the Talent Project of Anhui Agricultural University (Hefei, Anhui, China; YJ2013-6) and the National Natural Science Foundation of China (Beijing, China; 31200107).

\section{REFERENCES}

Aguilar, B., B. Amorena, and M. Iturralde. 2001. Effect of slime on adherence of Staphylococcus aureus isolated from bovine and ovine mastitis. Vet. Microbiol. 78:183-191.

AHVLA (Animal Health and Veterinary Laboratories Agency). 2001. Veterinary Investigation Surveillance Report. AHVLA, London, UK.

Amorena, B., E. Gracia, M. Monzón, J. Leiva, C. Oteiza, M. Pérez, J.L. Alabart, and J. Hernández-Yago. 1999. Antibiotic susceptibility assay for Staphylococcus aureus in biofilms developed in vitro. J. Antimicrob. Chemother. 44:43-55.

Baselga, R., I. Albizu, M. De La Cruz, E. Del Cacho, M. Barberan, and B. Amorena. 1993. Phase variation of slime production in Staphylococcus aureus: Implications in colonization and virulence. Infect. Immun. 61:4857-4862. 
Booth, J. M. 1997. Progress in mastitis control-An evolving problem. Pages 3-9 in Proc. Br. Mastitis Conf., Stoneleigh, UK. Institute of Animal Health, Compton, UK.

Campbell, I. M., D. N. Crozier, A. B. Pawagi, and I. A. Buivids. 1983. In vitro response of Staphylococcus aureus from cystic fibrosis patients to combinations of linoleic and oleic acids added to nutrient medium. J. Clin. Microbiol. 18:408-415.

Cramton, S. E., C. Gerke, N. F. Schnell, W. W. Nichols, and F. Götz. 1999. The intercellular adhesion (ica) locus is present in Staphylococcus aureus and is required for biofilm formation. Infect. Immun. 67:5427-5433.

Cramton, S. E., M. Ulrich, F. Götz, and G. Döring. 2001. Anaerobic conditions induce expression of polysaccharide intercellular adhesin in Staphylococcus aureus and Staphylococcus epidermidis. Infect. Immun. 69:4079-4085.

Cucarella, C., C. Solano, J. Valle, B. Amorena, I. Lasa, and J. R. Penadés. 2001. Bap, a Staphylococcus aureus surface protein involved in biofilm formation. J. Bacteriol. 183:2888-2896.

Fox, L. K., R. N. Zadoks, and C. T. Gaskins. 2005. Biofilm production by Staphylococcus aureus associated with intramammary infection. Vet. Microbiol. 107:295-299.

Götz, F. 2002. Staphylococcus and biofilms. Mol. Microbiol. 43:13671378.

Mack, D., N. Siemssen, and R. Laufs. 1992. Parallel induction by glucose of adherence and a polysaccharide antigen specific for plasticadherent Staphylococcus epidermidis: Evidence for functional relation to intercellular adhesion. Infect. Immun. 60:2048-2057.

McKenney, D., J. Hübner, E. Muller, Y. Wang, D. A. Goldmann, and G. B. Pier. 1998. The ica locus of Staphylococcus epidermidis encodes production of the capsular polysaccharide/adhesin. Infect. Immun. 66:4711-4720.

Melchior, M. B., H. Vaarkamp, and J. Fink-Gremmels. 2006. Biofilms: A role in recurrent mastitis infections? Vet. J. 171:398-407.
Oliveira, M., R. Bexiga, S. F. Nunes, C. Carneiro, L. M. Cavaco, F. Bernardo, and C. L. Vilela. 2006. Biofilm-forming ability profiling of Staphylococcus aureus and Staphylococcus epidermidis mastitis isolates. Vet. Microbiol. 118:133-140.

Peters, G., R. Locci, and G. Pulverer. 1981. Microbial colonization of prosthetic devices. II. Scanning electron microscopy of naturally infected intravenous catheters. Zentralbl. Bakteriol. Mikrobiol. Hyg. B 173:293-299.

Pozzi, C., E. M. Waters, J. K. Rudkin, C. R. Schaeffer, A. J. Lohan, P. Tong, B. J. Loftus, G. B. Pier, P. D. Fey, R. C. Massey, and J. P. O'Gara. 2012. Methicillin resistance alters the biofilm phenotype and attenuates virulence in Staphylococcus aureus device-associated infections. PLoS Pathog. 8:e1002626.

Rachid, S., K. Ohlsen, U. Wallner, J. Hacker, M. Hecker, and W. Ziebuhr. 2000. Alternative transcription factor $\sigma^{\mathrm{B}}$ is involved in regulation of biofilm expression in a Staphylococcus aureus mucosal isolate. J. Bacteriol. 182:6824-6826.

Takeuchi, S., T. Maeda, N. Hashimoto, K. Imaizumi, T. Kaidoh, and Y. Hayakawa. 2001. Variation of the agr locus in Staphylococcus aureus isolates from cows with mastitis. Vet. Microbiol. 79:267274.

Vasudevan, P., M. K. Nair, T. Annamalai, and K. S. Venkitanarayanan. 2003. Phenotypic and genotypic characterization of bovine mastitis isolates of Staphylococcus aureus for biofilm formation. Vet. Microbiol. 92:179-185.

Ziebuhr, W., C. Heilmann, F. Götz, P. Meyer, K. Wilms, E. Straube, and J. Hacker. 1997. Detection of the intercellular adhesion gene cluster (ica) and phase variation in Staphylococcus epidermidis blood culture strains and mucosal isolates. Infect. Immun. 65:890-896. 